\title{
Filtered Cigar
}

National Cancer Institute

\section{Source}

National Cancer Institute. Filtered Cigar. NCI Thesaurus. Code C127051.

A smaller cigar that is combined with a filter intended to reduce the amount of smoke, tar, and fine particles inhaled. 